\title{
Effect of Action Observation by Subject Type on the Balance and the Gait of Stroke Patients
}

\author{
Jong-Su Lee, PT, MS • Kyoung Kim, PT, $\mathrm{PhD}^{1 \dagger} \cdot$ Young-Mi Kim, PT, $\mathrm{PhD}^{2}$ \\ Department of Rehabilitation Science, Graduate School, Daegu University \\ ${ }^{1,2}$ Department of Rehabilitation Science, Graduate School, Daegu University
}

Received: September 18, 2018 / Revised: September 21, 2018 / Accepted: October 22, 2018

(C) 2019 J Korean Soc Phys Med

\section{| Abstract |}

PURPOSE: This study examined the effects of observing a self-video or a video of another person performing balance and gait training, followed by actual performance of the observed movements on the balance and walking ability of chronic stroke patients.

METHODS: Thirty patients, who had experienced a stroke and were admitted to $\mathrm{S}$ rehabilitation hospital for treatment, were selected randomly and divided into three groups with 10 patients each: self-action observation (SAO) group, other-action observation (OAO) group, and treadmill walking training (TWT) group. The training program was conducted five times per week for four weeks. The GAITRite system, $10 \mathrm{~m}$ walking test, and timed up and go test were performed to measure the subjects' gait and balance ability. RESULTS: The velocity, cadence, double support, and stride length were increased significantly in the SAO and OAO groups $(\mathrm{p}<.05)$ but the $\mathrm{T}$ group showed no significant

†Corresponding Author : Kyoung Kim

kykim257@hanmail.net, https://orcid.org/0000-0003-4169-6852

This is an Open Access article distributed under the terms of the Creative Commons Attribution Non-Commercial License (http://creativecommons.org/licenses/by-nc/3.0) which permits unrestricted non-commercial use, distribution, and reproduction in any medium, provided the original work is properly cited. changes; no significant difference was observed among the groups ( $p>05$ ). The 10MWT decreased significantly in the OAO group $(p<.05)$, but there were no significant changes in the SAO and T groups, and no significant difference was observed among the groups ( $\mathrm{p}>05)$. The TUG decreased significantly in the SAO and OAO groups $(\mathrm{p}<.05)$, but there were no significant changes in the $T$ group, and no significant difference was observed among the groups ( $\mathrm{p}>.05)$.

CONCLUSION: The self or other action observation training helps improve the balance and gait ability.

Key Words: Gait, Postural balance, Stroke

\section{Introduction}

The functional activity of stroke patients is reduced because post-stroke damage in the central nervous system and the motor and sensory nerve tracts lead to weakening of the upper and lower extremities and a delayed reaction time on the opposite side of the brain damage[1,2]. As a result, the overall motor control ability is reduced and the asymmetry of body weight support increases, resulting in a decreased balance control ability, decreased walking speed, and unstable gait pattern[3]. The most significant functional limitation after a stroke is gait disturbances, which is why patients regard independent and functional 
gait as the most important consideration[4]. To recover these damaged motor functions or acquire new motor functions, it is essential to change the plasticity of the brain neural network involved in the motor function. In addition, to promote neuroplasticity for restoring the motor function, it is necessary to utilize the visual, auditory, tactile, and proprioceptive senses appropriately in addition to exercise-based interventions[5]. Taking advantage of this information, the application of action observation training to rehabilitation was suggested recently.

Action observation training is a training method that can be performed independently by a patient without the help of a therapist The patient observes the movements of another person or video images and mimics the observed movements repeatedly[6]. Action observation training, which has a theoretical background of the mirror nervous system, has attracted significant attention since the publication of a report showing that the brain regions activated during action observation are the same as those activated during actual performance of the observed action because of the mirror nervous system[7]. On the other hand, previous studies on the effects of action observation in stroke patients focused mainly on the upper extremity function[8,9], and research on the lower extremity function is insufficient. This study examined whether there are any differences in the effects of training by observing a selfvideo of the patient and by observing a video of another person.

Therefore, this study examined the effects of observing a self-video or a video of another person performing balance and gait training, followed by actual performance of the observed movements on the balance and walking ability of chronic stroke patients.

\section{Methods}

\section{Participants}

Thirty patients, who were hospitalized in S rehabilitation hospital, were included in this study. The selection criteria for the study subjects were as follows: those who had suffered a stroke for more than six months; those who could walk more than $10 \mathrm{~m}$ independently or with the help of an assistive device; those who had no abnormality in their cognitive and visual function, as evidenced by a Korean Mini-Mental State Examination (MMSE-K) score of 23 or more; those who had no other neurological orthopedic diseases; and those who understood the purpose of the study and agreed to participate. Those who had limitations with communication and exercise learning due to unilateral neglect and moderate-to-severe aphasia were excluded.

The 30 patients selected were divided into three groups of 10 people each: self-action observation (SAO) group, other-action observation (OAO) group, and treadmill walking training (TWT) group. To minimize the selection bias, the subjects were divided randomly into the three groups. All the patients understood the purpose of this study and provided written informed consent prior to participation in accordance with the ethical principles of the Declaration of Helsinki.

\section{Research design}

The training program was conducted five times per week for four weeks. For three days before and after training, only evaluations were performed regardless of the groups. The experiment was continued for five weeks per group. For the evaluation items, their gait and balance ability was measured. The SAO group observed the movements in a self-video for 15 minutes per day and then practiced physical exercise of the same movements observed in the video for 15 minutes. The OAO group observed the movements of another person in a video for 15 minutes per day and then practiced physical exercise of the same movements observed in the video for 15 minutes. The TWT group performed treadmill walking training alone without action observation for 30 minutes per day. General physical therapy was applied to all three groups twice per day for 30 minutes. 


\section{Intervention}

Subjects in the SAO, OAO, and TWT groups participated in the program according to the designated schedule. The training was conducted in a quiet, independent space to exclude visual and auditory stimuli. To minimize the influence of contamination factors other than the designated training on the balance and gait ability, personal exercises of the lower limbs, except the general therapy and training of this study provided by the hospital program during the study participation period, were restricted in all subjects. For physical training and measurement, four physiotherapists with more than three years' experience worked together in this study, and were involved in the training of equipment usage, measurement methods, and training program's progress as well as in the practice before the start of each experiment and measurement to minimize the possible errors during the experimental process. Previous studies showed that the brain activity was increased more by actual practice of observed movements than by observations only[10] and that the practice and learning of the observed movements are important[11]. Therefore, in the present study, after the action observation training was completed, actual practice of the same movements observed in the video was carried out for the same time as the time spent in the action observation task. The SAO and OAO groups were included in this study to compare the effects of the observation of a self-video and of a video of another person. In addition, the TWT group was included as a control group to compare the difference between the action observation and the other walking training method. Therefore, a total of three groups were included in this study.

\section{1) SAO and OAO groups}

Sitting comfortably on a chair with armrests, the subject watched a monitor installed $1 \mathrm{~m}$ away playing a video of his/her own movements. To reduce the personal variations depending on the observation part, front- and lateral-side view videos of the paralyzed side were produced and provided. During viewing, the subjects were asked not to follow the contents in the video or move; the therapist encouraged the subject to concentrate on the contents of the video. The training program used in the present study, which was supplemented and modified from the action observation training program developed by Kim and Lee, was a four-step-training program composed of trunk stability training, lower extremity strength training, and balance and gait training. Each step was applied each week and the respective action observation training of each step was performed five times per week[12]. Step 1 consisted of a pelvic tilt exercise and a sit-to-stand exercise for trunk stability and lower limb muscle strength. Step 2 consisted of weight-shift training in the standing position for balance and gait. Step 3 consisted of gait training, and Step 4 consisted of obstacle and stair-walking training for the dynamic balance.

\section{2) TWT group}

The treadmill had a front instrument panel so that the speed could be adjusted according to the patient's walking ability. The speed could be adjusted from $0.1 \mathrm{kph}$ to 166 $\mathrm{kph}$ in $.1 \mathrm{kph}$ increments/decrements. Both sides of the treadmill had safety handles to allow the subject to grab with his/her hands if he/she lost balance during gait training, even though the subject was encouraged to move his/her arms freely without grabbing the safety handle with his/her hands. The training program consisted of gait training with different speeds without changing the angle of the ramp, at $1.5 \mathrm{kph}$ for weeks 1 and 2, and at $2.0 \mathrm{kph}$ for weeks 3 and 4.

\section{Outcome measures}

\section{1) GAITRite system}

The GAITRite system (CIR System Inc. USA) was used to analyze the spatial and temporal variables of gait. The 
subjects were instructed to stand $2 \mathrm{~m}$ away from a walking plate, and then walk on the walking plate $461 \mathrm{~cm}$ in length and $88 \mathrm{~cm}$ in width at their most comfortable walking speed according to a verbal signal of the examiner. The mean of three measurements was used for analysis. The gait data can be collected from both children and adults, and this method has been used as a reliable measurement tool[13].

\section{2) $10 \mathrm{~m}$ walking test (10 MWT)}

This test is used to assess the walking ability and the risk of falls; the inter-examiner reliability is .97[14]. The time spent walking $10 \mathrm{~m}$ of a $13 \mathrm{~m}$ distance excluding the first $1.5 \mathrm{~m}$ and the last $1.5 \mathrm{~m}$ at a maximum speed was measured three times, and the mean value of the three measurements was used for the analysis.

\section{3) Timed up and go (TUG)}

This test was a highly validated test method that is used to assess the dynamic balance, walking speed, and functional movement, and the inter-examiner reliability of this test is $.99[15]$. The time that a subject spent rising from a chair with armrests, walking to a target $3 \mathrm{~m}$ away and walking back, and sitting back in the chair was measured three times, and the mean of three measurements was used for the analysis.

\section{Statistical analysis}

The patients' characteristics, including age, height, weight, and disease period, were analyzed using one-way ANOVA. The pre- and post-intervention data were analyzed using a paired t-test for within-group differences and an analysis of the covariance for between-group differences. The analysis of the covariance was also used to control for any variance in the pre-intervention values. The pre-intervention value, group, and post-intervention value was considered as a covariate, independent variable, and dependent variable, respectively. All statistical analyses were performed using PAWS 20.0 (SPSS Inc., Chicago, IL, USA), and $\mathrm{p}<.05$ was considered significant.

\section{Results}

A homogeneity test of the general characteristics of the subjects conducted before the experiment showed no significant differences in average age, weight, height, or muscle mass between the three groups ( $\mathrm{p}>$.05) (Table 1).

The velocity, cadence, double support, and stride length increased significantly in the SAO and OAO groups $(\mathrm{p}<.05)$, but there was little change in the TWT group; no significant difference was observed among the groups ( $>$.05). Single support, step length, and BOS were similar in the three groups $(\mathrm{p}>.05)$. The 10 MWT decreased significantly in the OAO group $(\mathrm{p}<.05)$, but there was no significant change in the SAO and TWT groups; no significant difference was observed among the groups ( $\mathrm{p}$ $>.05$ ). The TUG decreased significantly in the SAO and OAO groups $(\mathrm{p}<.05)$, but there was no significant change

Table 1. General Characteristics of the Subjects.

\begin{tabular}{ccccc}
\hline Category & SAO $(\mathrm{n}=10)$ & OAO $(\mathrm{n}=10)$ & TWT $(\mathrm{n}=10)$ & $\mathrm{p}$ \\
\hline Age $(\mathrm{yr})$ & $56.23 \pm 5.59$ & $59.91 \pm 7.21$ & $58.1 \pm 6.69$ & .378 \\
Height $(\mathrm{cm})$ & $165.53 \pm 8.87$ & $163.33 \pm 8.83$ & $160.40 \pm 5.89$ & .336 \\
Weight $(\mathrm{kg})$ & $65.38 \pm 4.73$ & $61.5 \pm 11.26$ & $63.90 \pm 12.60$ & .616 \\
Disease Period $(\mathrm{m})$ & $17.54 \pm 11.25$ & $20.42 \pm 12.20$ & $23.90 \pm 13.42$ & .473 \\
\hline
\end{tabular}

SAO: Self-action observation group

OAO: Other-action observation group

TWT: Treadmill walking training group 
Table 2. Mean and Standard Deviation (SD) of the Each Group

\begin{tabular}{|c|c|c|c|c|}
\hline & & Pre-value & post-value & $\mathrm{p}$ \\
\hline \multirow{9}{*}{$\mathrm{SAO}(\mathrm{n}=10)$} & velocity $(\mathrm{cm} / \mathrm{sec})$ & $47.28 \pm 16.68$ & $56.24 \pm 22.06$ & $.001 *$ \\
\hline & cadence (steps/min) & $77.02 \pm 11.89$ & $82.49 \pm 15.08$ & $.009^{*}$ \\
\hline & single support $(\%)$ & $.39 \pm .09$ & $.36 \pm .08$ & .141 \\
\hline & double support $(\%)$ & $.53 \pm .16$ & $.44 \pm .15$ & $.000^{*}$ \\
\hline & step length (cm) & $38.27 \pm 11.54$ & $41.40 \pm 10.56$ & .136 \\
\hline & stride length $(\mathrm{cm})$ & $66.15 \pm 15.67$ & $71.95 \pm 18.30$ & $.0113^{*}$ \\
\hline & BOS $\left(\mathrm{cm}^{2}\right)$ & $13.57 \pm 3.46$ & $15.21 \pm 3.95$ & .076 \\
\hline & 10MWT(sec) & $15.44 \pm 6.29$ & $13.84 \pm 5.74$ & .070 \\
\hline & TUG(sec) & $22.97 \pm 14.01$ & $17.84 \pm 10.04$ & $.007 *$ \\
\hline \multirow{9}{*}{ OAO $(n=10)$} & velocity $(\mathrm{cm} / \mathrm{sec})$ & $42.21 \pm 28.01$ & $51.88 \pm 32.61$ & $.021 *$ \\
\hline & cadence (steps/min) & $74.83 \pm 16.00$ & $82.48 \pm 14.33$ & $.005 *$ \\
\hline & single support $(\%)$ & $.32 \pm .08$ & $.34 \pm .04$ & .307 \\
\hline & double support $(\%)$ & $.48 \pm .21$ & $.39 \pm .17$ & $.001 *$ \\
\hline & step length (cm) & $38.09 \pm 19.72$ & $40.50 \pm 20.67$ & .266 \\
\hline & stride length $(\mathrm{cm})$ & $58.02 \pm 24.77$ & $65.79 \pm 26.26$ & $.008^{*}$ \\
\hline & $\operatorname{BOS}\left(\mathrm{cm}^{2}\right)$ & $16.39 \pm 5.46$ & $17.06 \pm 7.08$ & .499 \\
\hline & 10MWT (sec) & $23.83 \pm 18.46$ & $20.72 \pm 17.98$ & $.023 *$ \\
\hline & TUG (sec) & $27.50 \pm 22.68$ & $23.11 \pm 17.48$ & $.044 *$ \\
\hline \multirow{9}{*}{ TWT $(n=10)$} & velocity $(\mathrm{cm} / \mathrm{sec})$ & $34.98 \pm 15.39$ & $46.96 \pm 30.38$ & .091 \\
\hline & cadence (steps/min) & $76.97 \pm 18.05$ & $80.29 \pm 21.09$ & .252 \\
\hline & single support $(\%)$ & $.35 \pm .06$ & $.38 \pm .09$ & .297 \\
\hline & double support $(\%)$ & $.78 \pm .48$ & $.73 \pm .50$ & .662 \\
\hline & step length (cm) & $32.57 \pm 11.29$ & $35.44 \pm 15.14$ & .340 \\
\hline & stride length $(\mathrm{cm})$ & $60.10 \pm 26.12$ & $66.31 \pm 28.01$ & .125 \\
\hline & BOS $\left(\mathrm{cm}^{2}\right)$ & $15.38 \pm 4.52$ & $15.46 \pm 5.06$ & .948 \\
\hline & 10MWT (sec) & $29.81 \pm 19.41$ & $24.74 \pm 14.86$ & .189 \\
\hline & TUG (sec) & $32.58 \pm 18.30$ & $27.83 \pm 18.32$ & .201 \\
\hline
\end{tabular}

SAO: Self-action observation group

OAO: Other-action observation group

TWT: Treadmill walking training group

* significant difference between pre- and post-test $(\mathrm{p}<.05)$.

Single support, Step length : Affected side

in the TWT group, and no significant difference was observed among the groups ( $\mathrm{p}>.05)$ (Table 2).

\section{Discussion}

Action observation training combines observation and exercise, thereby increasing the excitability of the cortical spinal tract[16]. Its neurophysiological effects and impact on the activation of brain regions have been demonstrated through brain imaging studies, and action observation training has recently been shown to be effective in the rehabilitation of stroke patients[17].

This study examined the effects of the action observation of video images, followed by physical training by performing the actual practice of the observed movements on balance and gait among the various rehabilitation programs to improve the balance and gate ability of stroke patients. In addition, either a self-video or a video of another person was provided for observation to compare the difference in the effects from the control group. 
Table 3. Outcomes of ANCOVA in the Post Intervention

\begin{tabular}{ccccc}
\hline & SAO $(\mathrm{n}=10)$ & OAO $(\mathrm{n}=10)$ & TWT $(\mathrm{n}=10)$ & $\mathrm{p}$ \\
\hline velocity $(\mathrm{cm} / \mathrm{sec})$ & $49.93 \pm 3.74$ & $51.65 \pm 3.84$ & $55.46 \pm 4.28$ & .629 \\
cadence (steps/min) & $81.74 \pm 2.09$ & $83.89 \pm 2.19$ & $79.59 \pm 2.39$ & .425 \\
single support $(\%)$ & $.35 \pm .02$ & $.36 \pm .02$ & $.39 \pm .02$ & .310 \\
double support $(\%)$ & $.48 \pm .06$ & $.47 \pm .06$ & $.58 \pm .07$ & .454 \\
step length $(\mathrm{cm})$ & $39.81 \pm 2.16$ & $39.08 \pm 34.50$ & $39.22 \pm 2.48$ & .969 \\
stride length $(\mathrm{cm})$ & $67.36 \pm 2.43$ & $69.47 \pm 2.52$ & $67.87 \pm 2.74$ & .832 \\
BOS $\left(\mathrm{cm}^{2}\right)$ & $16.67 \pm .94$ & $15.76 \pm .97$ & $15.14 \pm 1.05$ & .555 \\
\hline 10MWT $(\mathrm{sec})$ & $19.41 \pm 1.73$ & $19.61 \pm 1.73$ & $18.86 \pm 1.96$ & .959 \\
TUG (sec) & $21.09 \pm 1.82$ & $22.94 \pm 1.87$ & $23.82 \pm 2.07$ & .600 \\
\hline
\end{tabular}

SAO: Self-action observation group

OAO: Other-action observation group

TWT: Treadmill walking training group

The results revealed significant changes in the speed, cadence, double support, and stride length of the GAITRite system, and the TUG for both the SAO and OAO groups after training. The 10MWT changed significantly after training only in the OAO group. No significant difference was observed between the groups. Consistent with the findings of the present study, a previous study reported no significant differences between the self-versus non-selfhand movement observation[18]. They supported the idea that mirror neurons can process visual information on the actions of both the self and others in a similar way[19].

In addition, the findings of the present study were similar to those of a previous study showing that action observation training improved the dynamic balance, walking speed, and cadence. Moreover, the training increased the single limb support time of the paralyzed lower limb[12]. Similar results were obtained in a study of 22 stroke patients, in which action observation training and general gait training were applied to the experimental and control group, respectively. The experimental group showed significant differences in their cadence, gait velocity, stride, step, single limb support, double limb support, stride length, and step length. The control group showed significant differences only in their stride[20]. These results suggest that action observation training showing the gait of a normal adult can help patients imitate and memorize the appropriate muscle control through repetitive exercise training, producing a new motion circuit[21], and achieving a gait pattern close to that of a normal person

The study conducted by Hiyamizu et al. in young adults showed different results from the findings of the present study. In their study, balance training on a flat surface was applied to a group that performed actual practice following the observations of another person's movements, to a group that performed actual practice following the action observation of a self-video, and to a group that performed only the practice without observation. Only the group that combined the actual practice with the action observation of a self-video showed an increase in balance[22].

Similar to the abovementioned studies, in the present study, the balance and walking elements were improved after the patients underwent action observation training. A previous study reported that motion memory was formed after action observation followed by actual practice of the observed video, whereas motion memory was not formed in the group that performed the same movements but 
observed different videos from the practiced movements [23]. Similarly, in the present study, repeated observation and practice of the movements of oneself or another person were likely to result in motion learning by activating mirror nerve cells. The physical training of performing the same movements after the observation added the motion practice element to the action observation, thereby enhancing the recovery of the damaged region and having a greater impact on the recovery of the patients' abnormal brain activity and motor function.

To summarize the results of the present study and those of previous studies, it is concluded that action observation training is effective for motion imitation and practice. This study had several limitations. Extreme care should be taken when attempting to generalize these findings to the entire stroke patient population because a small sample size was used in this study, no comparative analysis of different brain damage areas was carried out, and no direct identification of the brain activation site was performed. Therefore, it will be necessary to standardize the videos for action observation through further studies.

\section{Conclusion}

Action observation training by showing a video of themselves or another person helped improve the patients' balance and gait ability. If applied in practice, this treatment is expected to increase the therapeutic effect by allowing the patients to watch a video in the waiting room before receiving therapy.

\section{References}

[1] Andrews AW, Bohannon RW. Distribution of muscle strength impairments following stroke. Clin Rehabil. 2000; 14(1):79-87.

[2] Bellelli G, Buccino G, Bernardini B, et al. Action observation treatment improves recovery of postsurgical orthopedic patients: evidence for a top-down effect? Arch Phys Med Rehabil. 2010;91(10):1489-94.

[3] Celnik P, Webster B, Glasser DM, et al. Effects of action observation on physical training after stroke. Stroke. 2008;39(6):1814-20.

[4] Duncan P, Studenski S, Richards L, et al. Randomized clinical trial of therapeutic exercise in subacute stroke. Stroke. 2003;34(9):2173-80.

[5] Franceschini M, Agosti M, Cantagallo A, et al. Mirror neurons: action observation treatment as a tool in stroke rehabilitation. Eur J Phys Rehabil Med. 2010;46(4): $517-23$.

[6] Hamilton AF, Grafton ST. Action outcomes are represented in human inferior frontoparietal cortex. Cereb Cortex. 2008;18(5):1160-8.

[7] Haslinger B, Erhard P, Altenmuller E, et al. Transmodal sensorimotor networks during action observation in professional pianists. J Cogn Neurosci. 2005;17(2): 282-93.

[8] Hesse S. Rehabilitation of gait after stroke: evaluation, principles of therapy, novel treatment approaches, and assistive devices. Top Geriatr Rehabil. 2003;19(2):109-26.

[9] Hiyamizu M, Maeoka H, Matsuo A, et al. Effects of self-action observation on standing balance learning: A change of brain activity detected using functional nearinfrared spectroscopy. NeuroRehabilitation. 2014; 35(3):579-85.

[10] Horstman AM, Beltman MJ, Gerrits KH, et al. Intrinsic muscle strength and voluntary activation of both lower limbs and functional performance after stroke. Clin Physiol Funct Imaging. 2008;28(4):251-61.

[11] Hyndman D, Ashburn A, Stack E. Fall events among people with stroke living in the community: circumstances of falls and characteristics of fallers. Arch Phys Med Rehabil. 2002;83(2):165-70.

[12] Johnson-Frey SH, Maloof FR, Newman-Norlund R, et al. Actions or hand-object interactions? Human inferior frontal cortex and action observation. Neuron. 2003; 
39(6):1053-8.

[13] Kaesler D, Mellifont R, Kelly PS, et al. A novel balance exercise program for postural stability in older adults: a pilot study. Journal of Bodywork and Movement Therapies. 2007;11(1):37-43.

[14] Kang K-Y. Effects of Observed Action Gait Training on Spatio-temporal Parameter and Motivation of Rehabilitation in Stroke Patients. Journal of the Korean Society of Physical Medicine. 2013;8(3):351-60.

[15] Kim JH, Lee BH. Action observation training for functional activities after stroke: a pilot randomized controlled trial. NeuroRehabilitation. 2013;33(4):565-74.

[16] Kwakkel G, van Peppen R, Wagenaar RC, et al. Effects of augmented exercise therapy time after stroke: a meta-analysis. Stroke. 2004;35(11):2529-39.

[17] Maranesi M, Livi A, Bonini L. Processing of own hand visual feedback during object grasping in ventral premotor mirror neurons. Journal of Neuroscience. 2015; 35(34): 11824-9.
[18] Menz HB, Latt MD, Tiedemann A, et al. Reliability of the GAITRite walkway system for the quantification of temporo-spatial parameters of gait in young and older people. Gait Posture. 2004;20(1):20-5.

[19] Patuzzo S, Fiaschi A, Manganotti P. Modulation of motor cortex excitability in the left hemisphere during action observation: a single-and paired-pulse transcranial magnetic stimulation study of self-and non-self-action observation. Neuropsychologia. 2003;41(9):1272-8.

[20] Rizzolatti G, Craighero L. The mirror-neuron system. Annu Rev Neurosci. 2004;27:169-92.

[21] Sakamoto M, Muraoka T, Mizuguchi N, et al. Executiondependent modulation of corticospinal excitability during action observation. Exp Brain Res. 2009; 199(1):17-25.

[22] Small SL, Buccino G, Solodkin A. The mirror neuron system and treatment of stroke. Dev Psychobiol. 2012; 54(3): 293-310.

[23] Tremblay C, Robert M, Pascual-Leone A, et al. Action observation and execution: intracranial recordings in a human subject. Neurology. 2004;63(5):937-8. 